九州大学学術情報リポジトリ

Kyushu University Institutional Repository

\title{
Vacuum-ultraviolet stimulated emission generated via four-wave Raman mixing in molecular hydrogen
}

Thang Phan Dinh

Division of International Strategy, Center of Future Chemistry, Kyushu University

Vu, Duong

Division of International Strategy, Center of Future Chemistry, Kyushu University

Imasaka, Totaro

Division of International Strategy, Center of Future Chemistry, Kyushu University

http://hdl. hand le. net/2324/2800477

出版情報: Applied physics. B, Lasers and optics. 125 (128), 2019-06-19. Springer Nature バージョン：

権利関係 : 


\section{Vacuum-ultraviolet stimulated emission generated via four-wave Raman mixing in molecular hydrogen}

Thang Phan Dinh ${ }^{1,2} \cdot$ Duong $\mathrm{Vu}^{1,3} \cdot$ Totaro Imasaka ${ }^{1,4^{*}}$

${ }^{1}$ Division of International Strategy, Center of Future Chemistry, Kyushu University, 744 Motooka, Nishiku, Fukuoka 819-0395, Japan

${ }^{2}$ Center for Laser Technology, National Center for Technological Progress, Ministry of Science and Technology, C6 Build, Thanh Xuan North, Thanh Xuan District, Hanoi, Vietnam

${ }^{3}$ Institute of Physics, Vietnam Academy of Science and Technology, 10, Dao Tan, Ba Dinh, Hanoi, Vietnam

${ }^{4}$ Hikari Giken, Co., 2-10-30, Sakurazaka, Chuou-ku, Fukuoka 810-0024, Japan

* Totaro Imasaka

TotaroImasaka@gmail.com 


\section{Abstract}

A two-color femtosecond pump beam (800 and $1200 \mathrm{~nm}$ ) and a one-color femtosecond probe beam $(267 \mathrm{~nm})$ were focused into a hydrogen gas to generate high-order Raman sidebands in the spectral region of $172-343 \mathrm{~nm}$. The efficiency of frequency conversion to neighboring Stokes/anti-Stokes emission was $18 \%$. Simultaneously, vacuum-ultraviolet stimulated emission was observed at 154.2 $\mathrm{nm}$ with the assistance of four-wave Raman mixing. This observation was explained as a Lyman transition $\left(B^{1} \Sigma_{\mathrm{u}}^{+} \rightarrow X^{1} \Sigma_{\mathrm{g}}^{+}\right)$of the hydrogen. 


\section{Introduction}

A variety of techniques have been developed for generating ultrashort optical pulses for spectroscopic and spectrometric studies in the ultraviolet (UV). For example, a deep-ultraviolet (DUV) femtosecond pulse has been generated at several wavelengths simultaneously and utilized in two-photon ionization mass spectrometry of organic molecules, such as pesticides, explosives, and nerve-agent metabolites in trace analyses [1]. A vacuum-ultraviolet (VUV) photon has sufficient energy for single-photon ionization; thus, a VUV femtosecond pulse would be a more versatile ionization tool.

In 1965, stimulated VUV emission was first proposed for the $C^{1} \Pi_{\mathrm{u}} \rightarrow X^{1} \Sigma_{\mathrm{g}}{ }^{+}$Werner transitions of molecular hydrogen in the 110-nm range [2]. In computer simulations, lasing was predicted in the range of 102.5-123.9 $\mathrm{nm}$ [3]. However, there are more electrons with sufficient energy to excite the $B\left(v^{\prime}=1-7\right)^{1} \Sigma_{\mathrm{u}}{ }^{+}$state rather than the $C^{1} \Pi_{\mathrm{u}}$ state in a discharge [2]. In 1970, sub-nanosecond VUV laser action was first observed for a series of Lyman bands based on the discrete transition $B\left(v^{\prime}\right)^{1} \Sigma_{\mathrm{u}}^{+} \rightarrow X\left(v^{\prime \prime}\right)$ ${ }^{1} \Sigma_{\mathrm{g}}{ }^{+}$in a fast electric discharge using a Blumlein parallel-plate [4,5]. Laser emissions appeared from $158.074 \mathrm{~nm}\left(v^{\prime}=7\right)$ to $161.318 \mathrm{~nm}\left(v^{\prime}=5\right)$ [4], and from $156.73 \mathrm{~nm}\left(v^{\prime}=8\right)$ to $157.18 \mathrm{~nm}\left(v^{\prime}=2\right)$, where $v^{\prime}$ was the vibrational quantum number in the upper state [5]. In 1978, it was theoretically predicted that stimulated VUV emission could be observed from the Lyman bands (122 - $170 \mathrm{~nm})$ by selective excitation, e.g., by optical pumping using the radiation from hydrogen arcs or energetic transitions in argon discharges [6]. However, it is essentially difficult to use a discharge technique for generating a femtosecond VUV pulse, thus making the application to femtosecond spectroscopy such as femtosecond time-resolved photoelectron spectroscopy difficult $[7,8]$.

There are several approaches for the generation of ultrashort VUV pulses. A $\mathrm{KBe}_{2} \mathrm{BO}_{3} \mathrm{~F}_{2}$ crystal has been used to generate harmonics down to $149.8 \mathrm{~nm}[9,10]$. This approach, however, suffers from phase mismatching below $200 \mathrm{~nm}$ because of the large group-velocity dispersion of the material. This restricted the pulse width to $100 \mathrm{fs}$, although a different type of material could generate shorter optical pulses. Another approach is four-wave mixing (FWM) in a noble gas such as argon or krypton or a solid material $[11,12]$. However, the conversion efficiency has been limited to $1 \%$ or less $[13,14]$. 
The efficiency of FWM can be significantly improved when a Raman transition is used, i.e., fourwave Raman mixing (FWRM). With molecular hydrogen as a medium, many Raman sidebands have been generated from the DUV to the near-infrared (NIR) region $[15,16]$. Hydrogen is one of the most efficient media for the Raman and produces the largest vibrational shift $\left(4155 \mathrm{~cm}^{-1}\right)$. With a two-color beam consisting of an 800-nm fundamental and a 1200-nm Stokes beam, which are separated by the Raman shift frequency $\left(4155 \mathrm{~cm}^{-1}\right)$, Raman sidebands were generated to the 9th-order anti-Stokes emission (200 nm) [17-19]. The conversion efficiency to neighboring anti-Stokes emissions approached $27 \%$.

For efficient generation of Raman sidebands in the DUV-VUV region, a pump-probe technique has been developed whereby hydrogen was resonantly excited by FWRM to its first vibrational level by an $800-, 1200-\mathrm{nm}$ two-color pump beam. The coherent vibration of the hydrogen frequencymodulated a probe beam, producing high-order Stokes and anti-Stokes sidebands in the vicinity of the probe wavelength. For a 267- or 200-nm probe beam, high-order anti-Stokes beams were generated down to $185 \mathrm{~nm}$ [17-19]. However, the efficiency of the high-order Raman generation was limited by anomalous dispersion and thus significant phase mismatching in the DUV-VUV region.

It is suggested using a different type of optical configuration, referred to as BOXCARS (crossedbeam, phase-matched, coherent anti-Stokes Raman scattering spectroscopy), for better phase matching, whereby three beams consisting of two-color pump and one-color probe beams are non-collinearly focused into the Raman medium. This approach has been successfully utilized for efficient generation of high-order anti-Stoke Raman sidebands in a solid material such as a diamond crystal having large dispersion [20].

In this study, high-order Raman sidebands were generated via FWRM by focusing a two-color (800-nm, 1200-nm) femtosecond pump beam and a 267-nm femtosecond probe beam into molecular hydrogen, as shown in Fig. 1. By using a BOXCARS optical configuration shown in Fig. 2 (A), antiStoke emission was generated down to $172 \mathrm{~nm}$ (fifth order). Simultaneously, stimulated VUV emission was observed at $154.2 \mathrm{~nm}$, which was explained as a Lyman transition from the $B^{1} \Sigma_{\mathrm{u}}{ }^{+}$ electronic excited state prepared by FWRM to the $X^{1} \Sigma_{\mathrm{g}}{ }^{+}$ground state. 


\section{Materials and Methods}

\subsection{Pump beam}

Figure 2 (B) shows the scheme of the experimental setup used in this study. A fundamental beam of a Ti:sapphire laser ( $800 \mathrm{~nm}, 35 \mathrm{fs}, \Delta \lambda=16 \mathrm{~nm}, 3.6 \mathrm{~W}, 1 \mathrm{kHz}$, Legend Elite, Coherent) was divided into two beams with a beam splitter in an optical parametric amplifier (OPA, OPerA Solo, Coherent). The first beam $(17 \%, 610 \mathrm{~mW})$ was one component of the two-color pump beam. The other beam (83\%) was used by the OPA to generate a $1200-\mathrm{nm}$ Stokes beam ( $35 \mathrm{fs}, \Delta \lambda=100 \mathrm{~nm}, 450 \mathrm{~mW}$ ) for the second component of the two-color pump beam. The Stokes polarization was rotated with a half-wave plate (HWP) to be parallel to that of the fundamental beam. These beams were combined with a dichroic mirror (DM-2), and temporally overlapped with a delay stage (DL-1). The two-color beam was focused into a gas cell with a 750-mm-focal-length concave mirror (CM-1).

\subsection{Probe beam}

The remaining part $(1.6 \mathrm{~W})$ of the fundamental beam from the OPA was used for third harmonic generation of the probe beam. A BBO type I crystal (BBO-1) was used to generate the second harmonic and a BBO type II crystal (BBO-2) was used to generate the third harmonic by sumfrequency mixing the fundamental and second harmonic beams. A time plate (TP) was used to compensate for the time delay of the second harmonic beam relative to the fundamental beam, and a dual-wave plate (DWP, half-wave for $800 \mathrm{~nm}$, one-wave for $400 \mathrm{~nm}$ ) was used to adjust the polarizations. The third harmonic emission ( $39 \mathrm{fs}, \Delta \lambda=2.6 \mathrm{~nm}, 60 \mathrm{~mW}$ ) was isolated with a dichroic mirror (DM-1), the pulse width being determined with an in-house self-diffraction frequency-resolved optical gating system. The time delay of the probe pulse with respect to the two-color pump beam was adjusted with a delay stage (DL-2) to generate the maximum number of Raman sidebands. The third harmonic probe beam was focused into the gas cell with a 500-mm-focal-length concave mirror (CM2) and combined with the two-color pump beam with a dichroic mirror (DM-3). Note that the directions, the focal points, and the diameters at the focal points (beam divergences) should be 
carefully optimized. Otherwise, mismatching/misalignment decreases the efficiency in the generation of the Raman sidebands.

\subsection{Raman cell}

A Raman cell was filled with a hydrogen gas, the pressure of which was monitored with a pressure meter (AP44, Keyence). Calcium fluoride $\left(\mathrm{CaF}_{2}\right)$ windows on both sides of the cell were 0.5 -mm thick to minimize dispersion. The cell was directly connected to a vacuum chamber to avoid VUV absorption by air.

\subsection{Measurement of optical parameters}

The input powers of the $1200-\mathrm{nm}, 800-\mathrm{nm}$, and $267-\mathrm{nm}$ beams before entering the gas cell were 200 $\mathrm{mW}, 300 \mathrm{~mW}$, and $32 \mathrm{~mW}$, respectively, as measured with a power meter (FieldMate and FieldMaxIITO, Coherent). The DUV-VUV beam generated in the cell was focused by a 50 -cm-focal-length concave mirror (CM-3) and isolated by a dichroic mirror (DM-4). The emission spectrum was acquired either with a DUV spectrometer (DET-MAYAPRO-UV, 190-410 nm spectral range, 5- $\mu \mathrm{m}$ slit width, 0.16-nm spectral resolution, Ocean Optics), or a VUV spectrometer (DET-MAYAPRO-UV, 145-360 nm spectral range, 25- $\mu \mathrm{m}$ slit width, 0.26-nm spectral resolution, Ocean Optics). The VUV spectrometer was purged with nitrogen to avoid VUV absorption by air. The pulse width was not measured in this study, due to limited phase matching and light transmittance of a nonlinear optical crystal in the VUV region. The output power was not measured due to a low pulse energy of the VUV emission; it is difficult to calibrate the spectral response of the VUV spectrometer since no standard lamp is available in this spectral region. 


\section{Results and Discussion}

\subsection{Generation of Raman sidebands}

In FWRM, the Raman sideband intensity increases with the square of the sinc function [17]. Therefore, phase matching of $\Delta \boldsymbol{k}=2 \boldsymbol{k}_{\mathrm{F}}-\boldsymbol{k}_{\mathrm{S} 1}-\boldsymbol{k}_{\mathrm{AS} 1}=0$, where $\boldsymbol{k}_{\mathrm{F}}, \boldsymbol{k}_{\mathrm{S} 1}$, and $\boldsymbol{k}_{\mathrm{AS} 1}$ are the fundamental, Stokes, and antiStokes wave vectors, respectively, is required for efficient sideband generation. The cross angle between the beams is usually zero (i.e., a collinear configuration) for a low-pressure gas-phase Raman medium. Numerous NIR Raman sidebands can be generated with hydrogen gas in the collinear configuration [17]. However, a BOXCARS configuration provided better phase matching in the DUV-VUV region because of the large dispersion, although the length of interaction between the laser beam and the Raman medium decreases. Raman sidebands can be suppressed in the vicinity of the two-color NIR beam in the BOXCARS configuration because of poor phase matching arising from the cross-angled beams and the limited length of interaction. Thus, the molecular vibrational energy induced by the two-color pump beam can be preserved in the gas cell. As shown in Fig. 3, when a 267-nm probe beam was introduced into the interaction region, several Raman sidebands were generated at $241 \mathrm{~nm}, 219 \mathrm{~nm}$, and $201 \mathrm{~nm}$ for the first-, second-, and third-order anti-Stokes, and at $300 \mathrm{~nm}$ and $343 \mathrm{~nm}$ for the first- and second-order Stokes emissions. The density of the Raman medium increases at high hydrogen pressures, which is preferential for the generation of the Raman sidebands. The dispersion, however, increases at higher gas pressures, resulting in phase mismatching and suppressing the Raman sidebands in the collinear configuration [19]. Figure 4 shows the dependence of the 300-nm Stokes and 241-nm anti-Stokes intensities on the cross angle between the pump and probe beams. The sideband generation efficiency had a maximum of $18 \%$ at a $2.7-\mathrm{mrad}$ cross angle. The high-order anti-Stokes (e.g., $219 \mathrm{~nm})$ generation efficiency had a similar dependence, suggesting that Raman sidebands were generated through a cascade process. An efficiency of $27 \%$ was reported for only the two-color beam in the collinear configuration. The lower value $(18 \%)$ observed here could be attributed to mismatching/misalignment of the three beams and to larger phase mismatching in the UV region. 


\subsection{Generation of VUV emission}

Figure 5 shows the emission spectrum of the Raman sidebands acquired with the VUV spectrometer. Raman sidebands up to 5 th order $(172 \mathrm{~nm})$ were observed using the BOXCARS configuration. The ratio of the signal intensities for the 5th- and 4th-order Raman sidebands was $11 \%$, which was in reasonable agreement with the efficiency (18\%) measured for the low-order Raman sidebands (due to a large fluctuation of the signals arising from high-order nonlinear effect). No 6th-order anti-Stokes $(160 \mathrm{~nm})$ was detected. Instead, a signal larger than the 172-nm 5th-order was reproducibly observed at $154.2 \mathrm{~nm}$, which cannot be assigned to one of the Raman sidebands because the frequency of this emission is not an integer multiple of the vibrational Raman shift frequency $\left(4155 \mathrm{~cm}^{-1}\right)$. Since the distance between the Raman cell and the spectrometer is longer than $1 \mathrm{~m}$, no spontaneous emission can be detected. Thus, the VUV emission is directional and is suggested to be a stimulated emission. To investigate its origin, the dependence of the 186-nm, 172-nm, and 154.2-nm intensities on hydrogen pressure was characterized. As shown in Fig. 6, the thresholds and the maximum intensities were observed at $110 \mathrm{kPa}$ and in a narrow range of $140-165 \mathrm{kPa}$, respectively. Similar trends would occur for a nonlinear process requiring phase matching, suggesting that the 154.2-nm emission occurs from the upper state which is prepared by FWRM. The precise VUV transition energy of hydrogen has been measured by a discharge/fluorescence technique [6]. Several large fluorescence signals have appeared from bound-free transitions (in addition to several bound-bound transitions) at $160.79 \mathrm{~nm}$ for a transition of $B\left(v^{\prime}=7\right)^{1} \Sigma_{\mathrm{u}}^{+} \rightarrow X\left(v^{\prime \prime}=k^{\prime \prime}\right)^{1} \Sigma_{\mathrm{g}}{ }^{+}$and at $156.66 \mathrm{~nm}$ for a transition of $B\left(v^{\prime}=9\right)^{1} \Sigma_{\mathrm{u}}^{+}$ $\rightarrow X\left(v^{\prime \prime}=k^{\prime \prime}\right)^{1} \Sigma_{\mathrm{g}}{ }^{+}$where $k^{\prime \prime}$ represents a transition to a continuum of the lower state, suggesting a transition of $B\left(v^{\prime}=10\right){ }^{1} \Sigma_{\mathrm{u}}{ }^{+} \rightarrow X\left(v^{\prime \prime}=k^{\prime \prime}\right){ }^{1} \Sigma_{\mathrm{g}}{ }^{+}$at $154.59 \mathrm{~nm}$, which is close to $154.2 \mathrm{~nm}$. No other possible transition was found in the reference, although it is difficult to eliminate all other possibilities because the spectrum is complicated for molecular hydrogen in the VUV region. The line shape of the 154.2-nm emission observed in Fig. 5 (B) is slightly asymmetric, which can be explained by the congested fluorescence spectrum observed in this spectral region for molecular hydrogen [6]. Then, the stimulated emission observed at $154.2 \mathrm{~nm}$ would have appeared based on this transition with an 
assistance of the anti-Stokes Raman sidebands. A possible mechanism could be two-photon (186-nm + 172-nm) excitation to high vibrational levels of $B^{1} \Sigma_{\mathrm{u}}{ }^{+}$or $C^{1} \Pi_{\mathrm{u}}$ and subsequent one-photon (800nm) stimulated emission by introducing the $800-\mathrm{nm}$ pulse as a seed beam (see Fig. 7), and/or direct two-photon (201-nm + 201-nm) excitation because of the similar threshold (110 kPa) and maximal signal intensity (145-185 kPa; the data at $201 \mathrm{~nm}$ are not shown in Fig. 6). It should be noted that the pressure dependence of the emission intensity at $154.2 \mathrm{~nm}$ has a trend similar to the product of the intensities of AS4 $(186 \mathrm{~nm}) \times \operatorname{AS} 5(172 \mathrm{~nm})$ and/or AS3 $(201 \mathrm{~nm}) \times \operatorname{AS} 3(201 \mathrm{~nm})$ as shown in Fig. 6, suggesting that these two photons generated by FWRM are required for excitation. The energy separation between the dissociation limit and the continuum is small and can be negligible, then the calculated energy difference between $B\left(v^{\prime}=10\right){ }^{1} \Sigma_{\mathrm{u}}^{+}$and $X\left(v^{\prime \prime}=0\right){ }^{1} \Sigma_{\mathrm{g}}$ is $100963 \mathrm{~cm}^{-1}$. This process requires a 792.3-nm fundamental laser wavelength $\left(=100963 \mathrm{~cm}^{-1} / 2 \times 4\right)$, which is within the bandwidth of the Ti:sapphire laser used in this study $(800 \pm 14 \mathrm{~nm})$. A spacing between the $\Delta v^{\prime}=1$ levels at around the $v^{\prime}=10$ level is reported to be ca. $2 \mathrm{~nm}$ [6], which is larger than the spectral band width of the 35 -fs optical pulse (ca. $1 \mathrm{~nm}$ ). Thus, the other level such as $v^{\prime}=9$ or 11 would not be excited, producing only an emission line at $154.2 \mathrm{~nm}$.

\subsection{Potential application}

The spectral bandwidth of the 154.2-nm emission was $1.25 \mathrm{~nm}$, as shown in Fig. 5 (B). Thus, the transform-limited pulse width was 28 fs, which was comparable to the pulse widths of the Raman sidebands (ca. 35 fs) and would be confirmed by cross-correlation measurement in the VUV region [21]. This unexpected result suggests that a femtosecond pulse can be generated in the VUV region using a transition of molecular hydrogen. The broad emission bandwidth (continuum) was because of the unbound lower state of the transition (dissociative broadening). This situation is similar to excimer lasers, which are capable of generating/amplifying an ultrashort optical pulse by using an ultrashort pump or seed pulse. Assuming the efficiency of $18 \%$ for generating the higher-order Raman sideband and the flat spectral response in the VUV spectrometer, the pulse energy of the 154.2-hm emission can be estimated to $4 \mathrm{~nJ}$ that would be sufficient for spectroscopic/spectrometric applications. 
Accordingly, a revival technique of using a direct VUV transition by appropriate nonlinear optical pumping would have potential for use as a light source in a variety of femtosecond spectroscopies in the VUV regions.

Acknowledgments This research was supported by a Grant-in-Aid for Scientific Research from the Japan Society for the Promotion of Science [JSPS KAKENHI, Grant Number JP26220806]. 


\section{References}

1. A. Hamachi, T. Okuno, T. Imasaka, Y. Kida, T. Imasaka, Resonant and nonresonant multiphoton ionization processes in the mass spectrometry of explosives. Anal. Chem. 87, 3027-3031 (2015)

2. A. Bazhulin, I.N. Knyazev, G.G. Petrash, On the possibility of stimulated emission in the far ultraviolet. P. Zh. Eksp. Teor. Fiz. 48, 975 (1965) [Sov. Phys. -JETP 21, 649-650 (1965)]

3. A.W. Ali, A.C. Kolb, Hydrogen molecular vacuum ultraviolet laser theory. Appl. Phys. Lett. 13, 259-261 (1968)

4. R.T. Hodgson, Vacuum-ultraviolet laser action observed in the Lyman bands of molecular hydrogen. Phys. Rev. Lett. 25, 494-497 (1970)

5. R.W. Waynant, J.D. Shipman, Jr., R.C. Elton, A.W. Ali, Vacuum ultraviolet laser emission from molecular hydrogen. Appl. Phys. Lett. 17, 383-384 (1970)

6. H. Schmoranzer, R. Zietz, Observation of selectively excited continuous vacuum ultraviolet emission in molecular hydrogen. Phys. Rev. A 18, 1472-1475 (1978)

7. A. Stolow, A.E. Bragg, D.M. Neumark, Femtosecond time-resolved photoelectron spectroscopy. Chem. Rev. 104, 1719-1757 (2004)

8. P. Wernet, J. Gaudin, K. Godehusen, O. Schwarzkopf, W. Eberhardt, Femtosecond time-resolved photoelectron spectroscopy with a vacuum-ultraviolet photon source based on laser high-order harmonic generation. Rev. Sci. Instrum. 82, 063114 (2011)

9. T. Nakazato, I. Ito, Y. Kobayashi, X. Wang, C. Chen, S. Watanabe, Phase-matched frequency conversion below $150 \mathrm{~nm}$ in $\mathrm{KBe}_{2} \mathrm{BO}_{3} \mathrm{~F}_{2}$. Opt. Express 24, 17149-17158 (2016)

10. T. Kanai, T. Kanda, T. Sekikawa, S. Watanabe, T. Togashi, C. Chen, C. Zhang,, Z. Xu, J. Wang, Generation of vacuum-ultraviolet light below $160 \mathrm{~nm}$ in a KBBF crystal by the fifth harmonic of a single-mode Ti:sapphire laser. J. Opt. Soc. Am. B 21, 370-375 (2004)

11. T. Horio, R. Spesyvtsev, T. Suzuki, Generation of sub-17 fs vacuum ultraviolet pulses at $133 \mathrm{~nm}$ using cascaded four-wave mixing through filamentation in Ne. Opt. Lett. 39, 6021-6024 (2014) 
12. J.L. Silva, H.M. Crespo, R. Weigand, Generation of high-energy vacuum UV femtosecond pulses by multiple-beam cascaded four-wave mixing in a transparent solid. Appl. Opt. 50, 1968-1973 (2011)

13. M. Ghotbi, P. Trabs, M. Beutler, F. Noack, Generation of tunable sub- 45 femtosecond pulses by noncollinear four-wave mixing. Opt. Lett. 38, 486-488 (2013)

14. M. Ghotbi, M. Beutler, F. Noack, Generation of $2.5 \mu \mathrm{J}$ vacuum ultraviolet pulses with sub-50 fs duration by noncollinear four-wave mixing in argon. Opt. Lett. 35, 3492-3494 (2010)

15. T. Imasaka, S. Kawasaki, N. Ishibashi, Generation of more than 40 laser emission lines from the ultraviolet to the visible regions by two-color stimulated Raman effect. Appl. Phys. B 49, 389-392 (1989)

16. H. Kawano, Y. Hirakawa, T. Imasaka, Generation of more than 40 rotational Raman lines by picosecond and femtosecond Ti:sapphire laser for Fourier synthesis. Appl. Phys. B 65, 1-4 (1997)

17. K. Motoyoshi, Y. Kida, T. Imasaka, High-energy, multicolor femtosecond pulses from the deep ultraviolet to the near infrared generated in a hydrogen-filled gas cell and hollow fiber. Appl. Sci. 4, 318-330 (2014)

18. O. Shitamichi, T. Imasaka, High-order Raman sidebands generated from the near-infrared to ultraviolet region by four-wave Raman mixing of hydrogen using an ultrashort two-color pump beam. Opt. Express 20, 27959-27965 (2012)

19. D. Vu, N.T. Nghia, T. Imasaka, Generation of a femtosecond vacuum ultraviolet optical pulse by four-wave Raman mixing. Opt. Laser Technol. 88, 184-187 (2017)

20. M. Zhi, X. Wang, A.V. Sokolov, Broadband coherent light generation in diamond driven by femtosecond pulses. Opt. Express 16, 12139-12147 (2008)

21. M. Ghotbi, P. Trabs, M. Beutler, F. Noack, Generation of tunable sub-45 femtosecond pulses by noncollinear four-wave mixing, Opt. Lett. 38, 486-488 (2013). 


\section{Figure Captions}

Fig. 1 Scheme for the generation of Raman sidebands based on two-color FWRM and three-color FWRM.

Fig. 2 (A) BOXCARS configuration. The cross angle between the two pump beams was several degrees (fixed, not measured in this study), and the angle between the pump beams and the probe beam was changed from 0 to $20 \mathrm{mrad}$ (see Fig. 4). The Raman sidebands emit at small angles optimal for phase matching. (B) Experimental block diagram. M: mirror, CM-1,2,3: concave mirrors, DL-1,2: delay lines, DM-1,2,3,4: dichroic mirrors, BBO-1,2: $\beta-\mathrm{BaB}_{2} \mathrm{O}_{4}$, HWP: half-wave plate, DWP: dualwave plate, TP: time plate $\left(\alpha-\mathrm{BaB}_{2} \mathrm{O}_{4}\right)$.

Fig. 3 Spectrum of Raman sidebands generated with the BOXCARS configuration. The two NIR pump beams were nearly collinearly focused, and the DUV probe beam was focused at an angle of several mrad. The spectrum was recorded at 190-kPa hydrogen pressure, using the DUV spectrometer. S, Stokes; AS, anti-Stokes.

Fig. 4 Generation efficiencies for the Raman sidebands as a function of cross angles between the pump and probe beams. The wavelengths refer to first Stokes $(300 \mathrm{~nm})$, first anti-Stokes $(241 \mathrm{~nm})$, and second anti-Stokes $(219 \mathrm{~nm})$. The pump beams were nearly collinearly focused, and the hydrogen pressure was $190 \mathrm{kPa}$.

Fig. 5 (A) Spectrum of the Raman sidebands generated using the BOXCARS configuration and acquired at $165-\mathrm{kPa}$ hydrogen pressure with the VUV spectrometer. S, Stokes; AS, anti-Stokes. (B) Expanded spectrum at $154 \mathrm{~nm}$. The Raman sidebands are split by self phase modulation, resulting in irregular shapes in the Raman sidebands. The spectral response of the VUV spectrometer is not 
calibrated. However, the grating data suggests reduced sensitivity at longer wavelengths, leading to a lower signal intensity at S1.

Fig. 6 Dependence of the signal intensities for the VUV emission at $154.2 \mathrm{~nm}$ and the Raman sidebands on hydrogen pressure. Fourth anti-Stokes $186 \mathrm{~nm}$; fifth anti-Stokes, $172 \mathrm{~nm}$. The products of the intensities for AS4 and AS5 (circle) and AS3 and AS3 (triangular) are shown as broken lines, respectively.

Fig. 7 Energy level diagram for molecular hydrogen with possible origin of 154.2-nm emission. 


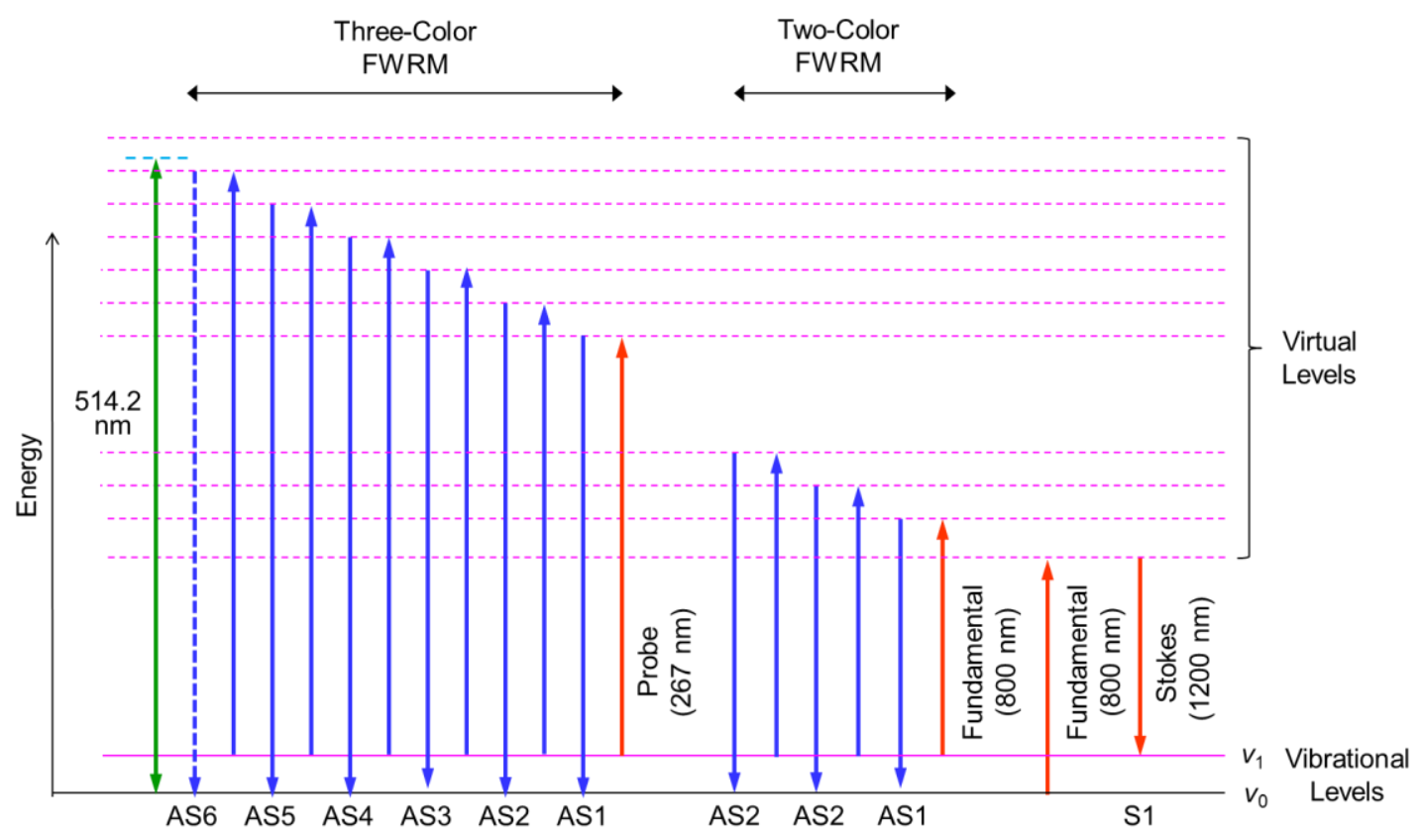

Figure 1 


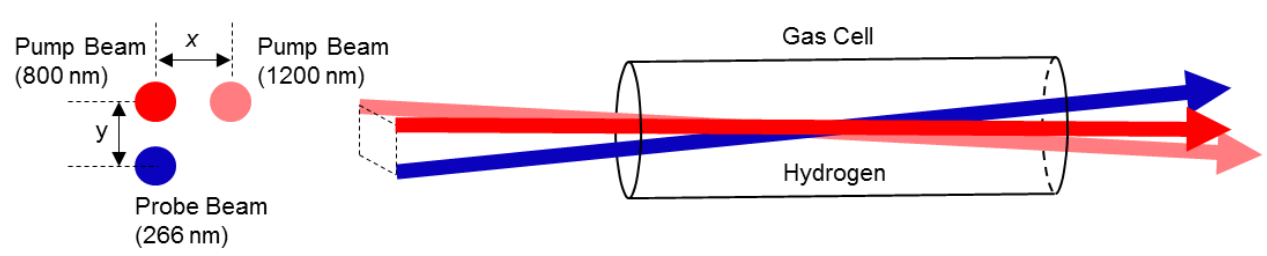

(A)

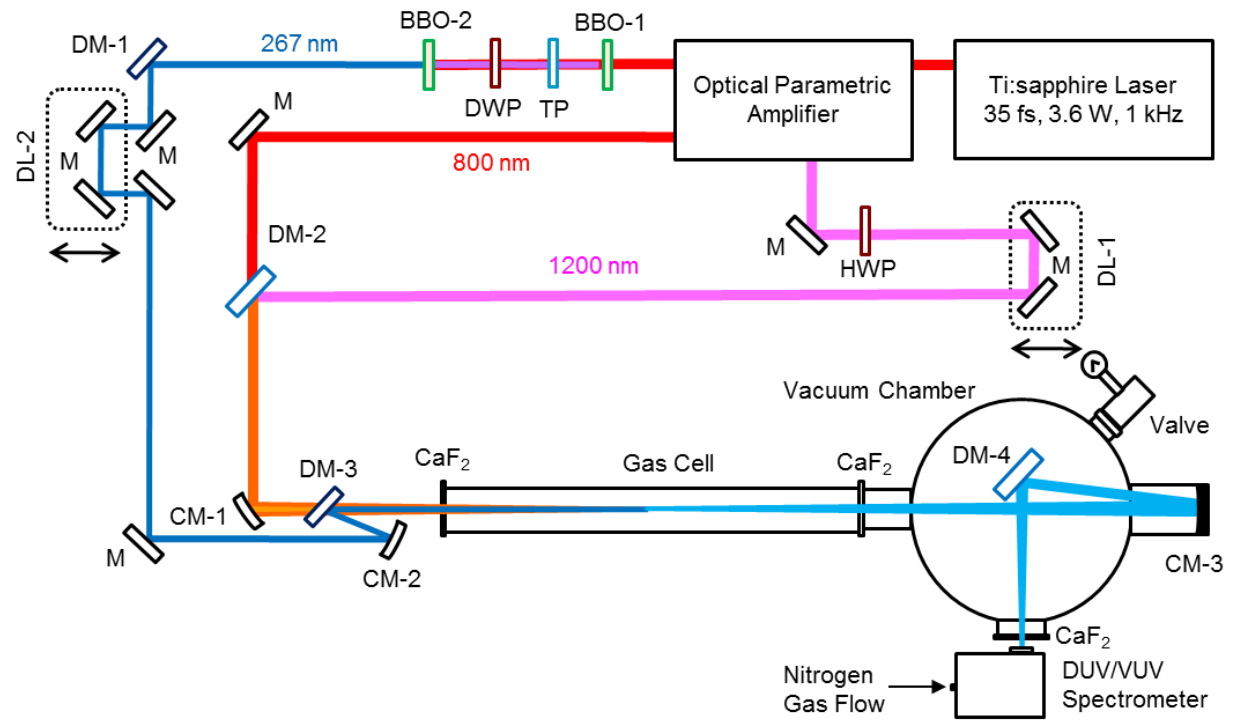

(B)

Figure 2 


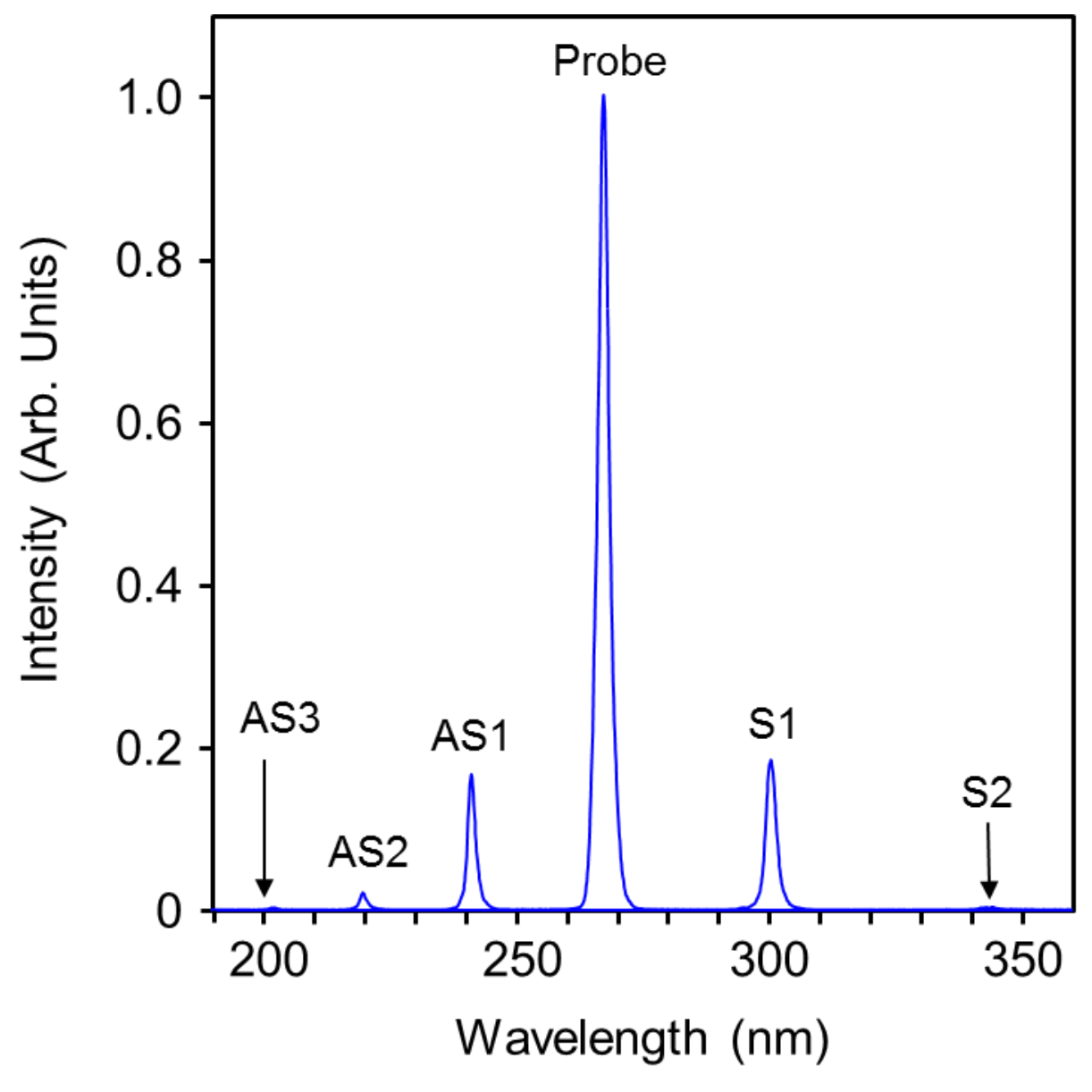

Figure 3 


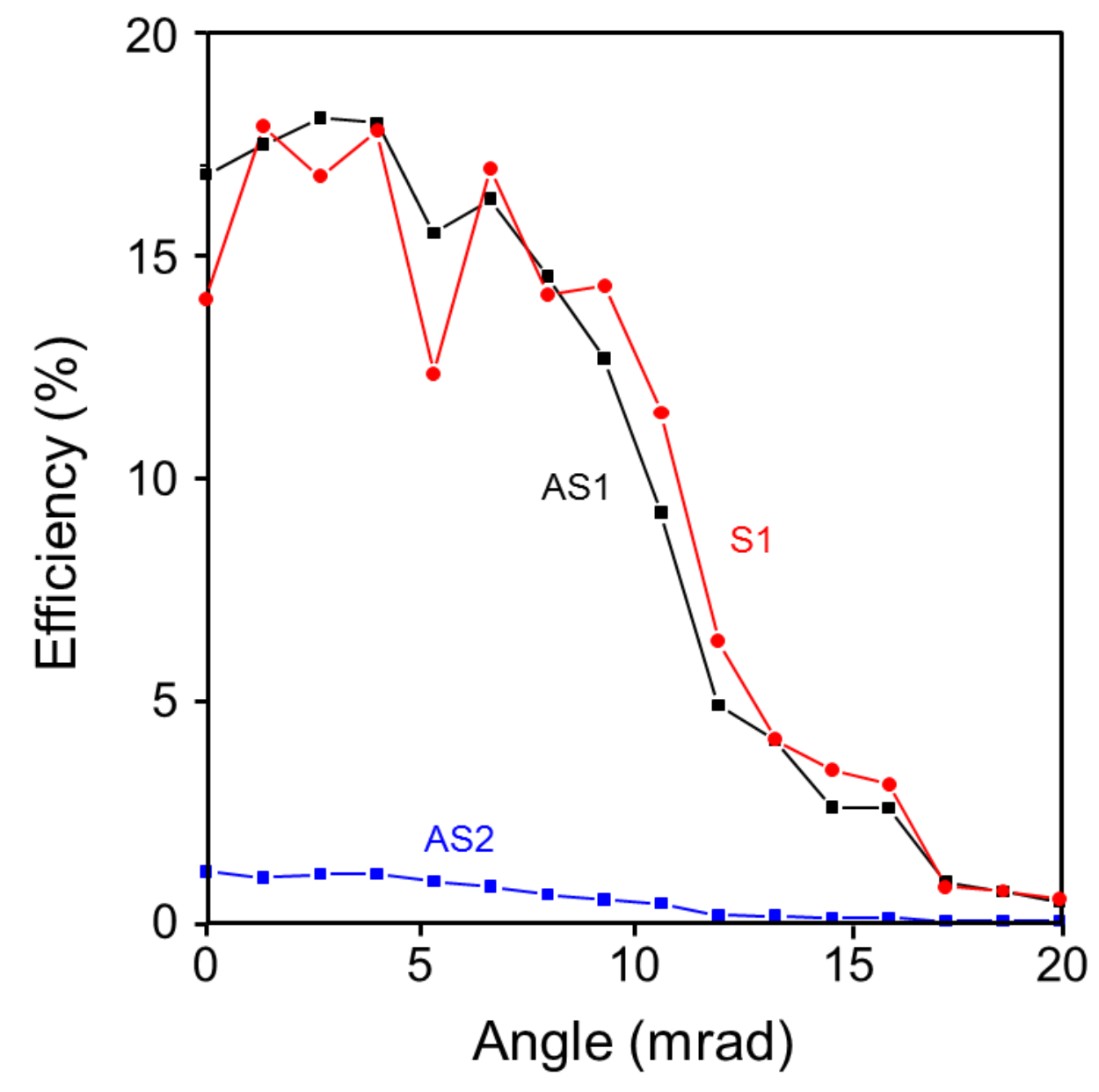

Figure 4 

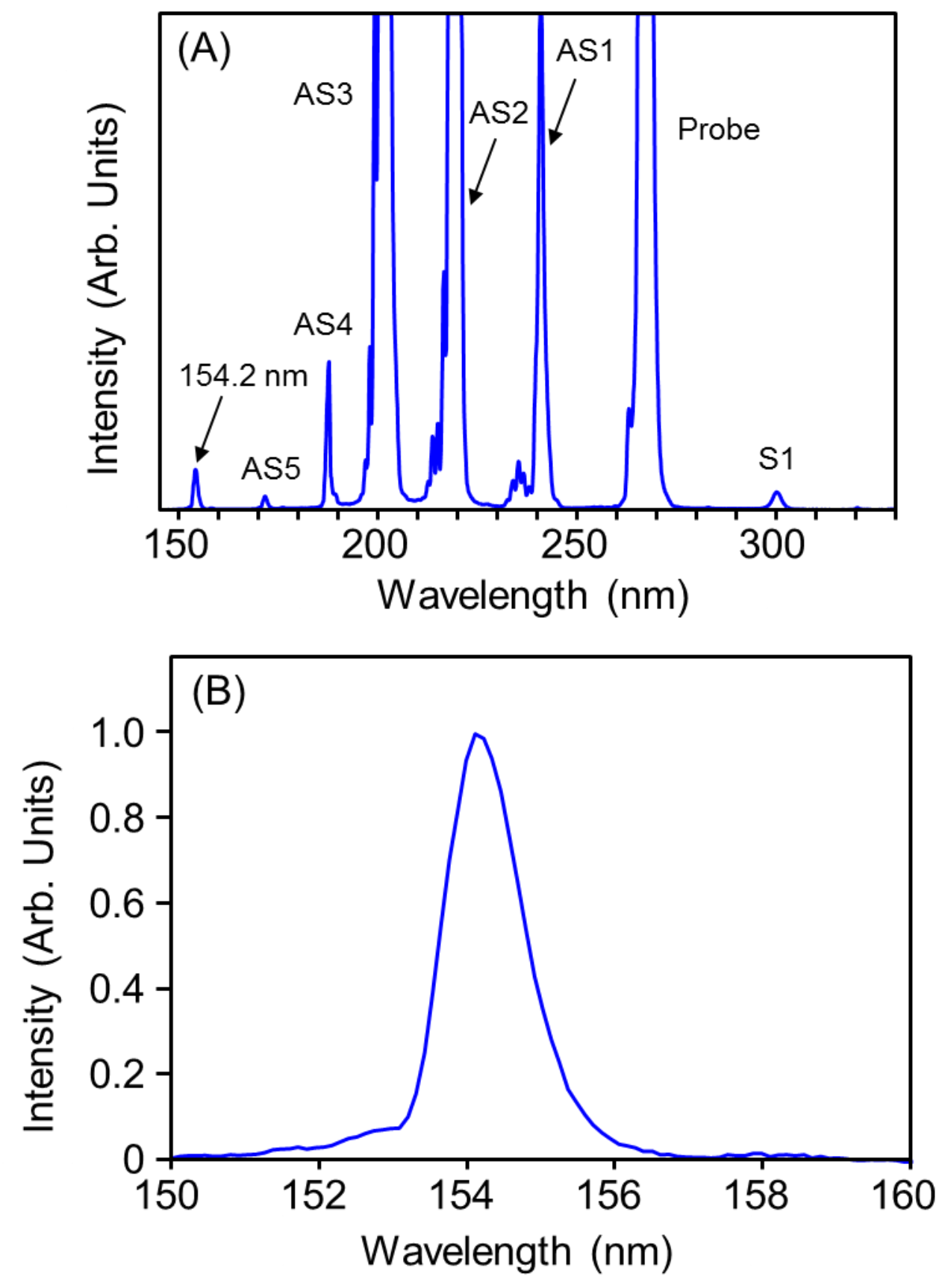

Figure 5 


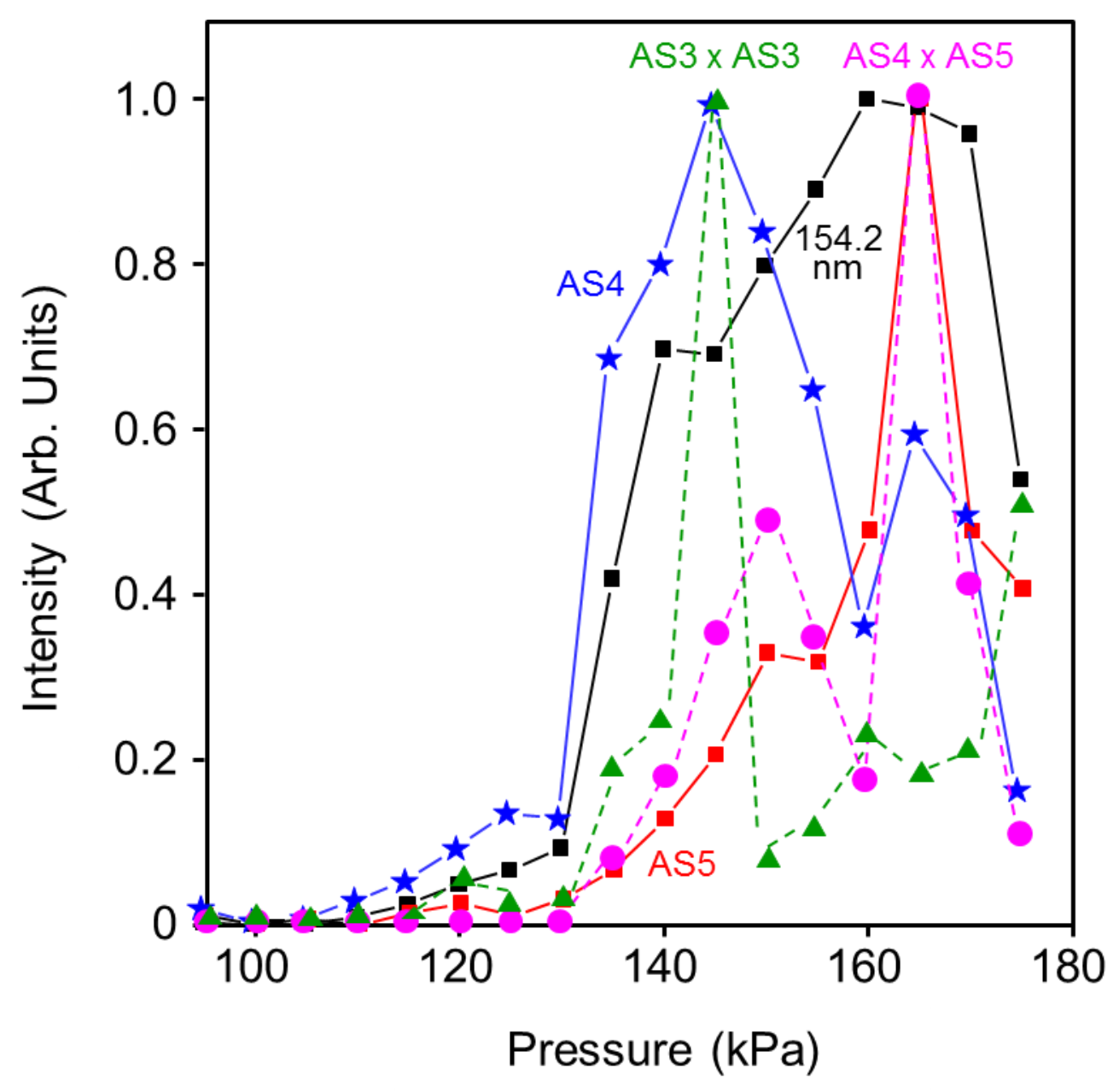

Figure 6 


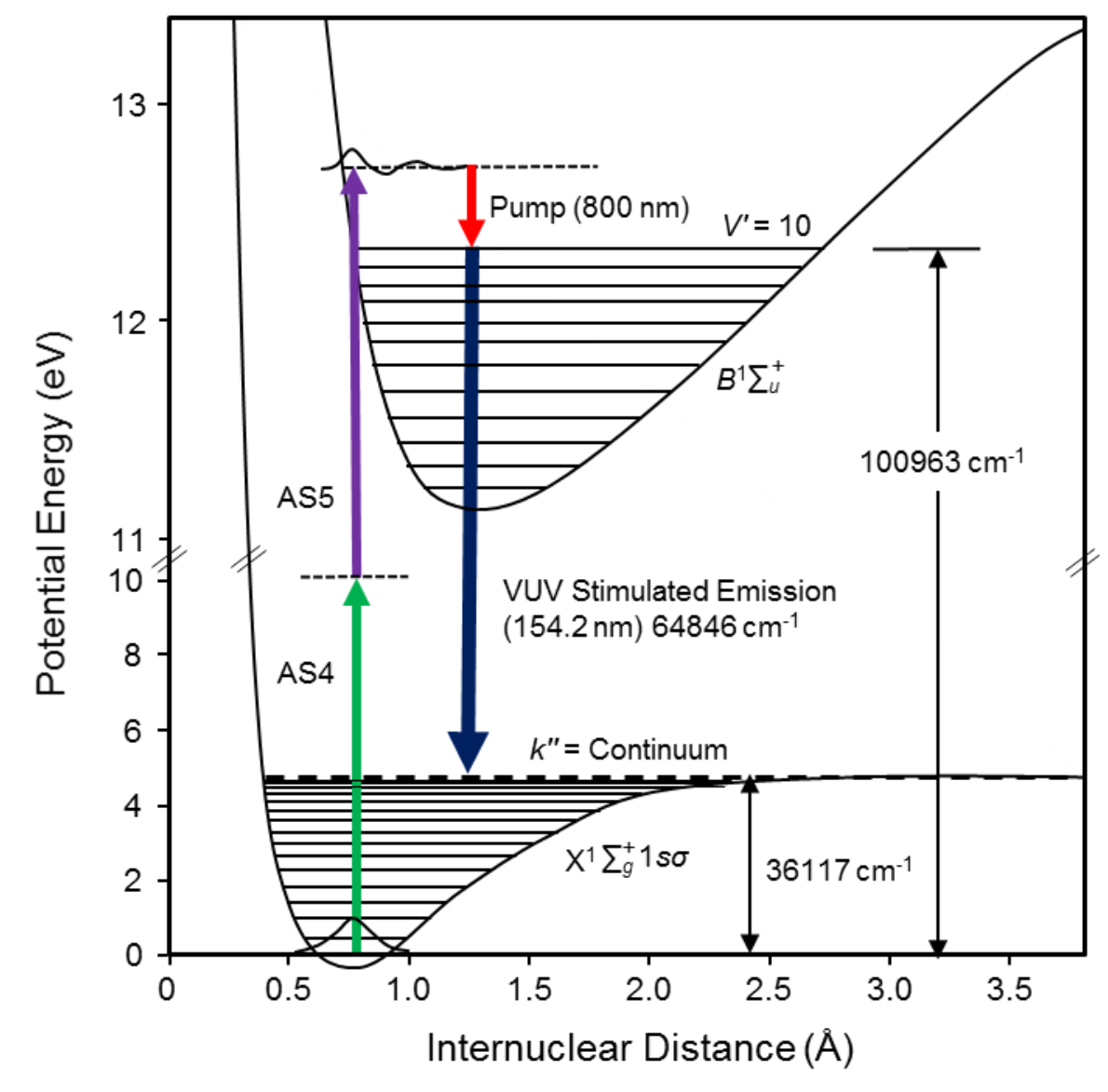

Figure 7 